\title{
INVESTIGATION OF HIGH ORDER STOCHASTIC DIFFERENTIAL EQUATIONS USING AVERAGING METHOD
}

\author{
Nguyen Dong Ani, Ngo Thi Hong Hue \\ Institute of Mechanics, VAST
}

\begin{abstract}
The averaging method is an useful tool for investigating both deterministic and stochastic quasilinear system. In the stochastic problems, however, the method has often been developed only for mechanical systems subjected to white noise excitations. In the paper this method is applied to high order stochastic differential equations. The nonlinear oscillations in high order deterministic differential equations were investigated in the fundamental work of Prof. Nguyen Van Dao. As an application of high order stochastic differential equations the nonlinear oscillation of single degree of freedom systems subjected to the excitation of a class of colored noises is outlined. The results obtained show that the higher order averaging method can also be successfully extended to the cases of colored noise excitation.
\end{abstract}

\section{INTRODUCTION}

Interest in the investigation of random phenomena is considerable over the recent years, due to various problems encountered in engineering applications. The well - known averaging method originally given by Krylov and Bogolibov and then developed by Mitropolskii is one of most popular methods for the approximate analysis of nonlinear systems [2]. The advantage of this method is that it reduces the dimension of the response coordinates. In the field of random vibration the averaging method was extended by Stratonovich [3] and has a mathematically rigorous proof by Khasminskii [4]. It is well-known, however, that the effect of some nonlinear terms is lost during first - order averaging procedure. In order to overcome this insufficiency, the procedures for obtaining higher approximate solutions in the stochastic averaging method were developed $[6-8,10]$. In the paper the averaging method is applied to high order stochastic differential equations. The nonlinear oscillations in high order deterministic differential equations were investigated in the fundamental work of Prof. Nguyen Van Dao [1].

Nonlinear random vibrations in dynamical systems subjected to the excitation of a white noise have often been investigated by many authors. It is well-known that the white noise process has a constant spectral density function and thus does not exits in the practice. Mean white, the random processes with linear - fractional spectral density function of the frequency describe well many real environmental loadings. These processes contain an important class of colored noises which can be interpreted as the result of the passage of white noise through a certain linear system with constant parameters, called a forming filter. As an application of high order stochastic differential equations the nonlinear oscillation of single degree of freedom systems subjected to the excitation of a class of colored noises is outlined [9]. 


\section{EXCITATION OF WHITE NOISE}

Let the dynamic system is described by a high order stochastic differential equation in the form $[1,5]$

$$
x^{(n)}+\alpha_{1} x^{(n-1)}+\ldots+\alpha_{n} x=\varepsilon F\left(t, x, \dot{x}, \ldots x^{(n-1)}\right)+\sqrt{\varepsilon} G\left(t, x, \dot{x}, \ldots, x^{(n-1)}\right) \dot{\xi}(t),
$$

where a dot denotes time differentiation and $x^{(k)}=\frac{d^{k} x}{d t^{k}}, \alpha_{I}$ are constants, $\varepsilon$ is a positive small parameter, $F$ and $G$ are nonlinear functions. The excitation $\dot{\xi}(t)$ is a zero mean Gaussian white noise stationary process with unit intensity

$$
E(\dot{\xi}(t) \dot{\xi}(t+\tau))=\delta(\tau)
$$

The operator $E$ denotes the mathematical expectation, $\delta(\tau)$ is Dirac - Delta function. The solution of the equation (2.1) depends on the roots of the characteristic equation of the generating linear equation corresponding to $(2.1)(\varepsilon=0)$

$$
L(\mu) \equiv \mu^{n}+\alpha_{1} \mu^{n-1}+\ldots+\alpha_{n-1} \mu+\alpha_{n}=0 .
$$

Suppose the characteristic equation (2.3) has $\mathrm{N}$ distinct double conjugate complex roots $\mu_{j}=\lambda_{j}-i \Omega_{j}, \bar{\mu}_{j}=\lambda_{j}+i \Omega_{j}, \lambda_{j}<0$, M distinct real negative roots $\mu_{2 N+k}=$ $h_{k}<0 ; H$ distinct double pure imagine roots $\mu_{2 N+M+l}=i \omega_{l}, \bar{\mu}_{2 N+M+l}=-i \omega_{l}$, where $\left|\lambda_{j}\right|,\left|h_{k}\right|, \Omega_{j}, \omega_{l}>>\varepsilon, j=\overline{1, N}, k=\overline{1, M}, l=\overline{1, H}, M+2 N+2 H=n$.

Using Ito formula for changing variables it can be shown that the solution of the equation (2.1) contains $M+2 N$ rapidly decaying components and $H$ slowly changing ones. Thus at the first approximation the rapidly decaying components can be neglected and the solution of the equation (2.1) is described by a $H$-frequency particular solution of the form:

$$
x(t)=\sum_{l=1}^{H} a_{l}(t) \cos \varphi_{l}, \quad \varphi(t)=\omega_{l} t+\theta_{l}(t)
$$

where $a_{l}(t), \theta_{l}(t)$ are determined by the system of stochastic differential equations [5]

$$
\begin{aligned}
& \frac{d a_{l}}{d t}=\varepsilon\left\{-\frac{F_{0} \sin \tilde{\varphi}_{l}}{\omega_{l}}+\frac{G_{0}^{2} \cos ^{2} \tilde{\varphi}_{l}}{2 a_{l} \omega_{l}^{2}}\right\}-\sqrt{\varepsilon} \frac{G_{0} \sin \tilde{\varphi}_{l}}{\omega_{l}} \dot{\xi}(t) \\
& \frac{d \theta_{l}}{d t}=\varepsilon\left\{-\frac{F_{0} \cos \tilde{\varphi}_{l}}{a_{l} \omega_{l}}-\frac{G_{0}^{2} \sin \tilde{\varphi}_{l} \cos \tilde{\varphi}_{l}}{a_{l}^{2} \omega_{l}^{2}}\right\}-\sqrt{\varepsilon} \frac{G_{0} \cos \tilde{\varphi}_{l}}{a_{l} \omega_{l}} \dot{\xi}(t)
\end{aligned}
$$


and it is denoted

$$
\begin{aligned}
& \left.\begin{array}{l}
F_{0}(a, \varphi, \theta)=F\left(t, x^{(p)}\right) \\
G_{0}(a, \varphi, \theta)=G\left(t, x^{(p)}\right)
\end{array}\right\} \text { with } x^{(p)}=\sum_{l=1}^{H} a_{l} \frac{\partial^{p}}{\partial t^{p}} \cos \varphi_{l}, \\
& a=\left(a_{1}, a_{2}, \ldots a_{H}\right), \quad \theta=\left(\theta_{1}, \theta_{2}, \ldots, \theta_{H}\right), \quad p=\overline{0, n-1} \\
& \tilde{\varphi}_{l}=\varphi_{l}+\varphi_{l}^{*}+\psi_{l}^{*}, \quad \varphi_{l}^{*}=\sum_{j=1}^{N} \varphi_{l j}^{*}, \quad \psi_{l}^{*}=\sum_{k=1}^{M} \psi_{l k}^{*}, \\
& \cos \varphi_{l j}^{*}=\frac{\lambda_{j}^{2}+\Omega_{j}^{2}-\omega_{l}^{2}}{R_{l j}}, \quad \sin \varphi_{l j}^{*}=-\frac{2 \omega_{l} \lambda_{j}}{R_{l j}}, \\
& \cos \psi_{l k}^{*}=-\frac{h_{k}}{r_{l k}}, \quad \sin \psi_{l k}^{*}=\frac{\omega_{l}}{r_{l k}}, \\
& R_{l j}=\sqrt{\left(\lambda_{j}^{2}+\Omega_{j}^{2}-\omega_{l}^{2}\right)+4 \omega_{l}^{2} \lambda_{j}}, \quad R_{l}=\prod_{j=1}^{N} R_{l j}, \\
& r_{l k}=\sqrt{h_{k}^{2}+\omega_{l}^{2}}, \quad r_{l}=\prod_{k=1}^{M} r_{l k} .
\end{aligned}
$$

To study the system (2.5), the corresponding Fokker - Planck equation for stationary probability density function $W(a, \theta)$ of amplitude and phase can be formed as follows

$$
\begin{aligned}
& \sum_{l=1}^{H}\left\{\frac{\partial}{\partial a_{l}}\left(K_{1 l} W\right)+\frac{\partial}{\partial \theta_{l}}\left(K_{2 l} W\right)-\frac{1}{2} \sum_{s=1}^{H} \frac{\partial^{2}}{\partial a_{l} \partial a_{s}}\left(H_{l s} W\right)\right. \\
& \left.-\frac{1}{2} \sum_{s=1}^{H} \frac{\partial^{2}}{\partial a_{l} \partial \theta_{s}}\left(N_{l s} W\right)-\frac{1}{2} \sum_{s=1}^{H} \frac{\partial^{2}}{\partial \theta_{l} \partial \theta_{s}}\left(M_{l s} W\right)\right\}=0
\end{aligned}
$$

where it is denoted

$$
\begin{aligned}
& K_{1 l}(a, \theta)=M_{t}\left\{-\frac{F_{0}(a, \varphi, t) \sin \tilde{\varphi}_{l}}{\omega_{l}}+\frac{G_{0}(a, \varphi, t) \cos \tilde{\varphi}_{l}}{2 a_{l} \omega_{l}^{2}}\right\}, \\
& K_{2 l}(a, \theta)=M_{t}\left\{-\frac{F_{0}(a, \varphi, t) \cos \tilde{\varphi}_{l}}{a_{l} \omega_{l}}-\frac{G_{0}^{2}(a, \varphi, t) \cos \tilde{\varphi}_{l} \sin \tilde{\varphi}_{l}}{a_{l}^{2} \omega_{l}^{2}}\right\}, \\
& R_{l s}(a, \theta)=M_{t}\left\{\frac{G_{0}^{2}(a, \varphi, t) \sin \tilde{\varphi}_{l} \sin \tilde{\varphi}_{s}}{\omega_{s} \omega_{l}}\right\}, \\
& N_{l s}(a, \varphi)=M_{t}\left\{\frac{G_{0}^{2}(a, \varphi, t) \cos \tilde{\varphi}_{l} \sin \tilde{\varphi}_{s}}{a_{l} \omega_{s} \omega_{l}}\right\}, \\
& M_{l s}(a, \theta)=M_{t}\left\{\frac{G_{0}^{2}(a, \varphi, t) \cos \tilde{\varphi}_{l} \cos \tilde{\varphi}_{s}}{a_{l} a_{s} \omega_{s} \omega_{l}}\right\} .
\end{aligned}
$$

The integration of the FP equation (2.7) is still a difficult problem. However, in some cases, the solution of (2.7) can be obtained in the explicit form [5].

\section{EXCITATION OF COLORED NOISE}

When a mechanical system subjected to colored noise the problem can be introduced to the high order differential equation subjected to white noise as considered in the previous section. In fact consider a mechanical system whose motion is described in the form:

$$
\ddot{x}+\omega^{2} x=\varepsilon f(x, \dot{x})+\sqrt{\varepsilon} q(t) .
$$


The excitation $q(x)$ is a normal stationary random process, the result of the passage of a white noise through the linear forming filter

$$
L q(t) \equiv \frac{d^{n}}{d t^{n}} q(t)+\sum_{s=0}^{n-1} \alpha_{s} \frac{d^{s}}{d t^{s}} q(t)=b \dot{\xi}(t),
$$

where $\alpha s, b=$ const, $\dot{\xi}(t)$ is a white noise of unit intensity. In the equation (3.1) $\omega$ is the natural frequency, $f$ is a nonlinear function of displacement and velocity. The spectral density of the process $q(t)$ can be easy obtained from (3.2)

$$
S_{q}(\omega)=\frac{1}{2 \pi} \frac{b^{2}}{l(i \omega) l(-i \omega)},
$$

where

$$
l(\lambda)=\lambda^{n}+\sum_{s=0}^{n-1} \alpha_{s} \lambda^{s}
$$

Eliminating now $q(t)$ from (3.1), (3.2) one obtains

$$
L\left(\ddot{x}+\omega^{2} x\right)=\varepsilon L f(x, \dot{x})+\sqrt{\varepsilon} b \dot{\xi}(t) .
$$

Thus, the equation (3.5) is of the form (2.1) and can be investigated using the method considered above.

For illustration let $q(t)$ be an exponentially correlated stationary random process, with following spectral density and correlation function

$$
S_{q}(\omega)=\frac{\delta_{1}^{2}}{\pi} \frac{a}{a^{2}+\omega^{2}}, K_{q}(\tau)=\delta_{1}^{2} e^{-\alpha|\tau|}, \sigma \gg \varepsilon .
$$

The corresponding forming filter is

$$
L q=\dot{q}(t)+\alpha q(t)=\delta_{1} \sqrt{2 \alpha} \dot{\xi}(t) .
$$

According to the procedure described the solution of the system (3.1), (3.7) is defined in the form:

$$
x(t)=a \cos \varphi, \quad \dot{x}=-a \omega \sin \varphi, \quad \ddot{x}=-a \dot{\omega}^{2} \cos \varphi,
$$

where $a(t), \theta(\mathrm{t})$ are Markov diffusion processes satisfying the equations

$$
\begin{gathered}
\dot{a}=\varepsilon u_{1}(a, \theta)+\sqrt{\varepsilon} v_{1}(a, \theta) \dot{\xi}(t) \\
\dot{\theta}=\varepsilon u_{2}(a, \theta)+\sqrt{\varepsilon} v_{2}(a, \theta) \dot{\xi}(t) \\
u_{1}(a, \varphi)=-\frac{(d f / d t+\alpha)_{0} \sin \left(\varphi+\varphi_{1}\right)}{\omega \sqrt{\alpha^{2}+\omega^{2}}}+\frac{\delta_{1}^{2} \alpha^{2} \cos ^{2}\left(\varphi+\varphi_{1}\right)}{\omega^{2} a\left(\omega^{2}+a^{2}\right)} \\
u_{2}(a, \varphi)=-\frac{(d f / d t+\alpha)_{0} \cos \left(\varphi+\varphi_{1}\right)}{\alpha \omega \sqrt{\alpha^{2}+\omega^{2}}}-\frac{\delta_{1}^{2} \alpha^{2} \sin ^{2}\left(\varphi+\varphi_{1}\right)}{\omega^{2} a^{2}\left(\omega^{2}+a^{2}\right)} \\
v_{1}(a, \varphi)=-\frac{\delta_{1} \sqrt{2 \alpha} \sin \left(\varphi+\varphi_{1}\right)}{\omega \sqrt{\alpha^{2}+\omega^{2}}}, \quad v_{2}(a, \varphi)=-\frac{\delta_{1} \sqrt{2 \alpha} \cos \left(\varphi+\varphi_{1}\right)}{\omega a \sqrt{\alpha^{2}+\omega^{2}}} \\
\sin \varphi_{1}=\frac{\omega}{\sqrt{\alpha^{2}+\omega^{2}}}, \quad \cos \varphi_{1}=\frac{\alpha}{\sqrt{\alpha^{2}+\omega^{2}}}
\end{gathered}
$$

Further, the solution to the corresponding FP equation can be performed as described above. 


\section{DUFFING OSCILLATOR SUBJECTED TO THE EXPONENTIALLY CORRELATED RANDOM PROCESS}

It is well-known that the exact solution of the Duffing oscillator subject to colored noise it not available up to now. So, the approximate solutions are to be interested. So, consider the Duffing system.

$$
\ddot{x}+2 \varepsilon h \dot{x}+\omega^{2} x+\varepsilon \gamma x^{2}=\sqrt{\varepsilon} q(t),
$$

where $q(t)$ is the exponentially correlated random process (3.6). For this case, one gets

$$
f(x, \dot{x})=-2 h \dot{x}-\gamma x^{3}, \quad \frac{d f}{d t}+\alpha f=-2 h \ddot{x}-2 \alpha h \dot{x}-3 \gamma x^{2} \dot{x}-\alpha \gamma x^{3} .
$$

Using the procedure described the second approximate probability density function of amplitude and phase to the Duffing system (4.1), (3.6) is found as:

$$
\begin{aligned}
W(a, \varphi)= & C a \exp \left\{\frac{-\left(\alpha^{2}+\omega^{2}\right) \omega^{2} h a^{2}}{\delta_{1}^{2} \alpha}\right\}\left\{1-\frac{3 \gamma \varepsilon}{16 \delta_{1}^{2}\left(a^{2}+\omega^{2}\right)} \times\right. \\
& \times\left[\gamma \omega^{2} a^{6}+\left(4 \alpha h \omega^{2}+h \alpha^{3}-\frac{h \omega^{4}}{\alpha}\right) a^{4}+\frac{6 \delta_{1}^{2}\left(\alpha^{2}-\omega^{2}\right) a^{2}}{\left(\alpha^{2}+\omega^{2}\right)}\right] \\
& +\frac{\varepsilon \gamma h \omega}{2 \delta_{1}^{2}} a^{4} \sin 2 \varphi-\left[\frac{3 \varepsilon \gamma}{2\left(\alpha^{2}+\omega^{2}\right)} a^{2}+\frac{\varepsilon \gamma h\left(\alpha^{2}-3 \omega^{2}\right)}{4 \delta_{1}^{2}} a^{4}\right] \cos 2 \varphi \\
& \left.+\frac{\varepsilon \gamma h \omega}{2 \delta_{1}^{2}} a^{4} \sin 4 \varphi-\left[\frac{3 \varepsilon \gamma}{4\left(\alpha^{2}+\omega^{2}\right)} a^{2}+\frac{3 \varepsilon \gamma h\left(\alpha^{2}-3 \omega^{2}\right)}{16 \delta_{1}^{2} \alpha} a^{4}\right] \cos 4 \varphi\right\} .
\end{aligned}
$$

In the limit case when the colored noise $q(t)$ tends to as white noise $\delta \dot{\xi}(t)$, i.e.

$$
\alpha, \delta_{1} \longrightarrow+\infty, \frac{2 \delta_{1}^{2}}{\alpha} \rightarrow \delta^{2}=\text { const }
$$

the solution (4.3) tends to the following expression

$$
W(a, \varphi)=C a \exp \left\{-\frac{2 \omega^{2} h a^{2}}{\delta^{2}}\right\}\left[1-\frac{\varepsilon \gamma h}{8 \delta^{2}} a^{4}(3+4 \cos 2 \varphi+\cos 4 \varphi)\right],
$$

which was obtained in [8].

Table 1. Mean - square amplitude of Duffing oscillation to colored noise for $\omega=1, \gamma=0.1 \mathrm{~h}=1 ; \alpha=0.1 ; \sigma_{1}^{2}=20$

\begin{tabular}{ccc}
\hline$\alpha$ & $\left\langle a^{2}\right\rangle$ & $\left\langle a^{2}\right\rangle_{0}$ \\
\hline 2.0 & 7.134 & 8.148 \\
4.9 & 4.500 & 4.719 \\
6.9 & 3.156 & 3.246 \\
8.0 & 2.143 & 2.462 \\
10.0 & 1.950 & 1.981 \\
\hline
\end{tabular}

In Tab.1 the mean - square amplitudes of Duffing oscillation subject to colored noise (3.6) are given when the bandwidth parameter $\alpha$ varies. It is seen that the mean - square 
amplitude decreases when $\alpha$ increases from 2.0 to 10.0 , and for $\alpha=10.0$ the mean - square amplitude is closed to the one (value 1.944) of the corresponding Diffing oscillator subject to the white noise with intensity equal to 0.4. In Tab.1 the mean - square amplitude of corresponding linear system $\left\langle a^{2}\right\rangle_{0},(y=0)$, is also given. It is shown that in the case of colored noise the effect of cubic non - linearity can be investigated by using higher order averaging procedure proposed. A similar result was also obtained for the Duffing oscillator (4.1) using the method of stochastic linearization $[11,12]$.

\section{CONCLUSION}

The averaging method is an useful tool for investigating both deterministic and stochastic quasilinear system. In the stochastic problems, however, the method has often been developed only for mechanical systems subjected to white noise excitations. In the paper this method is applied to high order stochastic differential equations. The nonlinear oscillations in high order deterministic differential equations were investigated in the fundamental work of Prof. Nguyen Van Dao. As an application of high order stochastic differential equations the nonlinear oscillation of single degree of freedom systems subjected to the excitation of a class of colored noises is outlined. The results obtained show that the higher order averaging method can also be successfully extended to the cases of colored noise excitation.

Acknowledgement. This publications is completed with financial support from the National Basic Research Program in Natural Science.

\section{REFERENCES}

1. Nguyen Van Dao, Nonlinear Oscillation of High Order Systems, NCSR of Vietnam, Hanoi, 1979.

2. Yu. A. Mitropolskii, Averaging Method in Nonlinear Mechanics, Naukova-Dumka, Kiev, 1971 (in Russian).

3. S. Stratonovich, Topics in the Theory of Random Noise, Gordon \& Breach v. 1, N. Y. 1963.

4. I. Gikhman, A. Skorokhod, Stochastic Differential Equations and Applications, Naukova Dumka, Kiev, 1982 (in Russian).

5. Yu. A. Mitropolskii, N. V. Dao, N. D. Anh, Nonlinear Oscillations in Systems of Arbitrary Order, Naukova - Dumka, Kiev, 1992 (in Russian).

6. Nguyen Dong Anh, Extended first order stochastic averaging method for a class of nonlinear systems, Vietnam Journal of Mathematics $21(3,4)(1993) 85$ - 93.

7. Nguyen Dong Anh, Higher approximate solutions on stochastic averaging method, Proc. of NCSR of Vietnam 5 (1) (1993) $19-26$.

8. Nguyen Dong Anh, Higher order averaging method of coefficients in Fokker-Planck equation, Indian Journal SADHANA 20 (Parts 2-4) (1995) 373 - 387.

9. N. D. Anh, Random oscillation in systems subjected to colored noises, Vietnam Journal of Mechanics 18 (1) (1996) 1-7.

10. Yu. A. Mitropolskii, N. D. Anh, N. D. Tinh, Random oscillations in the Van der Pol system under influence of wide-band stochastic process, Ucrainian Mathematical Journal 50 (11) (1998) 1517-1521, (in Russian).

11. Ngo Thi Hong Hue, Master Thesis, Institute of Mechanics, 1999 (in Vietnamese)

12. N. D. Anh, L. X. Hung, An Improved criterion of GEL for analysis of non-linear stochastic systems, J. Sound and Vibration 268 (2003) 177-200. 


\section{NGHIÊN CỨU PHƯƠNG TRÌNH VI PHÂN NGẪU NHIÊN CẤP CAO BẰNG PHƯƠNG PHÁP TRUNG BİNH}

Phương pháp trung bình là một công cụ tiện lợi trong việc nghiên cứu các hệ á tuyến tiền định và ngẫu nhiên. Tuy nhiên phương pháp này thường được áp dụng cho các hệ cơ học chịu kích động ồn trắng. Trong bài báo phương pháp trung bình đã được phát triên cho phương trình vi phân ngẫu nhiên cấp cao. Dao động phi tuyến trong các phương trình vi phân tiền định cấp cao đã được GS. Nguyễn Văn Đạo nghiên cứu một cách hệ thống bằng phương pháp tiệm cận. Một trong các ứng dụng của phương trình vi phân ngẫu nhiên cấp cao là cơ hệ chịu kích động ồn màu. Các kết quả nghiên cứu cho thấy phương pháp trung bình ngẫu nhiên bậc cao có thể áp dụng hiệu quả cho cơ hệ chịu kích động ồn màu. 